\title{
THE IMPORTANCE OF BEING ORLICZ
}

\author{
JÜRGEN APPELL \\ Mathematisches Institut, Universität Würzburg, Am Hubland, D-97074 Würzburg, Germany \\ E-mail: appell@mathematik.uni-wuerzburg.de
}

After Władysław Orlicz' pioneering invention in 1932 of the function spaces which now bear his name, a whole industry started dealing with the following problem: whenever there is an interesting result for Lebesgue spaces, try to formulate a parallel result for Orlicz spaces. In fact, it has become a quite useful device to use Orlicz spaces if one is led, for example, to nonlinear problems involving a nonlinearity of non-polynomial (e.g. exponential) growth.

The aim of this talk is to illustrate, by means of a series of examples, that extending results from the Lebesgue to the Orlicz space setting is by no means trivial or "automatic". These results include, but are not restricted to:

- examples and counterexamples in geometry of Banach spaces,

- operators which are unbounded and continuous, or bounded and discontinuous,

- differential equations with rapidly growing coefficients,

- integral equations with kernels of non-polynomial growth,

- singular integral equations with kernels of non-polynomial growth,

- imbeddings between Sobolev spaces with critical exponent.

In all these examples, it is important to point out that Orlicz spaces are used not just for technical reasons, but by the very nature of the problem involved. This also explains the somewhat cryptic title of the talk (after Oscar Wilde).

Recall that an Orlicz function (or Young function) is a positive, convex, even, and increasing (on $\mathbb{R}^{+}$) function $\Phi: \mathbb{R} \rightarrow \mathbb{R}$ satisfying

$$
\lim _{u \rightarrow 0} \frac{\Phi(u)}{u}=0, \quad \lim _{u \rightarrow \infty} \frac{\Phi(u)}{u}=\infty .
$$

Given a bounded domain $\Omega \subset \mathbb{R}^{N}$, the Orlicz space $L_{\Phi}=L_{\Phi}(\Omega)$ is defined by the

2000 Mathematics Subject Classification: Primary 46E30; Secondary 46E35, 47G10, 47H30.

The paper is in final form and no version of it will be published elsewhere. 
(Luxemburg) norm

$$
\|u\|_{\Phi}=\inf \left\{k>0: \int_{\Omega} \Phi\left(\frac{|u(x)|}{k}\right) d x \leq 1\right\} .
$$

Throughout the following, we will consider the three examples

$$
\Phi(u)=\frac{1}{p}|u|^{p}, \quad \Phi(u)=e^{|u|}-|u|-1, \quad \Phi(u)=e^{|u|^{p}}-1
$$

for $1<p<\infty$. The first Orlicz function in (1) leads to the classical Lebesgue spaces $L_{p}=L_{p}(\Omega)$ with norm

$$
\|u\|_{p}=\left(\int_{\Omega}|u(x)|^{p} d x\right)^{1 / p},
$$

the other two functions to more complicated Orlicz spaces. The main difference between the first and the other two Orlicz functions in (1) is that $\Phi(u)=|u|^{p}$ satisfies the $\Delta_{2^{-}}$ condition, but $\Phi(u)=e^{|u|}-|u|-1$ and $\Phi(u)=e^{|u|^{p}}-1$ don't.

Loosely speaking, one could say that the space $L_{\Phi}$ is not "too different" from the Lebesgue space $L_{p}$ if its Orlicz function $\Phi$ satisfies the $\Delta_{2}$-condition. (Some exceptions of this rule, however, occur in interpolation theory for linear operators, see [14].) To begin with, we illustrate this by means of some geometrical properties of the space $L_{\Phi}$. Given an Orlicz function $\Phi$, we denote as usual by $\tilde{\Phi}$ its complementary function

$$
\tilde{\Phi}(v)=\max _{u \geq 0}[u|v|-\Phi(u)] .
$$

For example, in case $\Phi(u)=\frac{1}{p}|u|^{p}$ we have $\tilde{\Phi}(v)=\frac{1}{\tilde{p}}|v|^{\tilde{p}}\left(\right.$ with $p^{-1}+\tilde{p}^{-1}=1$ ), and in case $\Phi(u)=e^{|u|}-|u|-1$ we have $\tilde{\Phi}(v)=(1+|v|) \log (1+|v|)-|v|$. The conjugate function of $\Phi(u)=e^{|u|^{p}}-1$ cannot be calculated explicitly.

Recall that the associate space (or Köthe dual) $\tilde{X}$ of a space $X$ of measurable functions is defined by the norm

$$
\|v\|_{\tilde{X}}=\sup _{\|u\|_{X} \leq 1} \int_{\Omega} u(x) v(x) d x .
$$

This is a (possibly strict) subspace of the usual dual space $X^{*}$, and $\tilde{X}=X^{*}$ if and only if $X$ is regular, i.e., every element in $X$ has an absolutely continuous norm. For example, $\tilde{L}_{p}=L_{p}^{*}$ for $1 \leq p<\infty$ but $\tilde{L}_{\infty} \subset L_{\infty}^{*}$.

THEOREM 1. The associate space of $L_{\Phi}$ is $\tilde{L}_{\Phi}=L_{\tilde{\Phi}}$. Moreover, the following four conditions are equivalent:
(a) $L_{\Phi}$ is regular;
(b) $L_{\Phi}$ is separable;
(c) $L_{\Phi}^{*}=L_{\tilde{\Phi}}$;
(d) $\Phi \in \Delta_{2}$.

Finally, $L_{\Phi}$ is reflexive if and only if both $\Phi \in \Delta_{2}$ and $\tilde{\Phi} \in \Delta_{2}$.

Theorem 1 shows that, while the Lebesgue space $L_{p}$ is always regular, separable, and reflexive for $1<p<\infty$, for the Orlicz space $L_{\Phi}$ all these properties heavily depend on growth properties of $\Phi$.

Our next example is concerned with the simplest nonlinear operator. Given a function of two variables $f: \Omega \times \mathbb{R} \rightarrow \mathbb{R}$, the operator $F$ defined by

$$
F u(x)=f(x, u(x))
$$


is called the Nemytskij operator (or superposition operator) generated by $f$. This operator occurs everywhere in nonlinear analysis, it suffices to think of the right-hand side of a differential equation.

The following remarkable theorem on the "automatic continuity" of the operator (2) is due to Krasnosel'skij [12]:

TheOREM 2. Suppose that the operator $F$ maps $L_{p}$ into $L_{q}$ for some $p, q \in[1, \infty)$. Then $F$ is continuous and bounded. In particular, $F$ is always continuous and bounded in case $F(C) \subseteq L_{q}$, where $C=C(\bar{\Omega})$ denotes the set of all continuous real functions on $\bar{\Omega}$.

From Theorem 2 it follows, in particular, that continuity and boundedness of the operator $F$ are in fact equivalent between Lebesgue spaces. So, from this point of view, $F$ behaves rather like a linear operator.

If one passes from Lebesgue to Orlicz spaces, the situation changes drastically. Indeed, the following two examples [13] show that in this case the operator (2) may be bounded and discontinuous, or continuous and unbounded.

Suppose first that $\Phi \in \Delta_{2}$, but $\Psi \notin \Delta_{2}$, and consider the function $f: \mathbb{R} \rightarrow \mathbb{R}$ defined by

$$
f(u):=\Psi^{-1}(\Phi(u)) .
$$

Then $F: L_{\Phi} \rightarrow L_{\Psi}$ is discontinuous at 0 , but bounded on every bounded set $M \subset L_{\Phi}$. Conversely, suppose now that $\Phi \notin \Delta_{2}$, but $\Psi \in \Delta_{2}$, and define $f$ again as in (3). Then $F: L_{\Phi} \rightarrow L_{\Psi}$ is unbounded on every ball of radius $r>1$, but continuous on the whole space $L_{\Phi}$.

The choice of the corresponding Young functions to satisfy or not the $\Delta_{2}$-conditions in these examples is not accidental. In fact, the following two theorems [13] show that the "automatic continuity" of $F$ depends only on the target space $L_{\Psi}$, while the "automatic boundedness" of $F$ depends only on the source space $L_{\Phi}$; compare this with Theorem 2:

TheOREM 3. Suppose that the operator $F$ maps $L_{\Phi}$ into $L_{\Psi}$, where $\Psi \in \Delta_{2}$. Then $F$ is continuous.

TheOREM 4. Suppose that the operator $F$ maps $L_{\Phi}$ into $L_{\Psi}$, where $\Phi \in \Delta_{2}$. Then $F$ is bounded.

Combining the two examples and theorems above, one could summarize these results in a somewhat sloppy way with the two equivalences

$$
F: L_{\Phi} \rightarrow L_{\Psi} \text { continuous } \Leftrightarrow \Psi \in \Delta_{2}, \quad F: L_{\Phi} \rightarrow L_{\Psi} \text { bounded } \Leftrightarrow \Phi \in \Delta_{2} .
$$

Of course, this is a completely new phenomenon which in Lebesgue spaces cannot occur. We point out that, in case of a "degenerate" function $f(x, u)=g(x) h(u)$, the operator $(2)$, i.e., $F u(x)=g(x) h(u(x))$, is always continuous from $C$ into $L_{\Psi}$, even if $\Psi \notin \Delta_{2}$. So, a parallel counterexample as (3) is not possible in this case. However, in case of a general function $f$ this is not true. For example [1], let $\Psi(u)=e^{|u|}-|u|-1$ and $f(x, u)=\log (x+|u|)$. Then the corresponding Nemytskij operator (2) maps $C$ into $L_{\Psi}$, but is discontinuous at 0 . 
Let us now pass to studying the linear integral operator

$$
K u(x)=\int_{\Omega} k(x, y) u(y) d y \quad(x \in \Omega)
$$

between Lebesgue and Orlicz spaces. Combining acting, continuity, and boundedness conditions for the operators (2) and (4) one may obtain existence results for the nonlinear Hammerstein equation

$$
\int_{\Omega} k(x, y) f(y, u(y)) d y=v(x) \quad(x \in \Omega) .
$$

To give sufficient (and sometimes even necessary) acting conditions for the operator (4) between the spaces $L_{p}$ and $L_{q}(1 \leq p, q \leq \infty)$, one usually considers the kernel classes

$$
[k]_{p, q}=\|x \mapsto\| k(x, \cdot)\left\|_{\tilde{p}}\right\|_{q}, \quad[k]_{p, q}^{*}=\|y \mapsto\| k(\cdot, y)\left\|_{q}\right\|_{\tilde{p}} .
$$

These classes are usually called Hilbert-Schmidt classes in case $p=q=2$ and HilleTamarkin classes in the general case. It is well-known (see, e.g., [7]) that the operator (4) maps $L_{p}$ into $L_{q}$ (and is then automatically continuous, by Banach's classical theorem $[5])$, if

$$
\min \left\{[k]_{p, q},[k]_{p, q}^{*}\right\}<\infty .
$$

Moreover, in this case the left-hand side of (5) gives an upper bound for the norm of $K$ between $L_{p}$ and $L_{q}$. One may also show that the condition $k \in L_{r}(\Omega \times \Omega)$ is sufficient for (5), where either $r=\tilde{p} q=p q /(p-1)$ or $r=\max \{\tilde{p}, q\}=\max \{p /(p-1), q\}$.

If one tries to get a parallel result between the Orlicz spaces $L_{\Phi}$ and $L_{\Psi}$, one has to consider kernel classes of the type $[k]_{\Phi, \Psi}=\|x \mapsto\| k(x, \cdot)\left\|_{\tilde{\Phi}}\right\|_{\Psi}$. However, it is simpler to imitate the special cases described above. To this end, we recall that the relation $\Phi \preceq \Psi$ between two Orlicz functions $\Phi$ and $\Psi$ means that $\Phi(u) \leq \Psi(k u)\left(|u| \geq u_{0}\right)$ for some $k>0$ and $u_{0}>0$. Then one gets the following sufficient conditions [13]:

TheOREm 5. Suppose that $k \in L_{\Xi}(\Omega \times \Omega)$, where either $\Psi \circ \tilde{\Phi} \preceq \Xi$, or $\tilde{\Phi} \circ \Psi \preceq \Xi$, or $\Psi \preceq \Xi, \tilde{\Phi} \preceq \Xi$ and $\tilde{\Xi} \in \Delta^{\prime}$. Then the operator (4) maps $L_{\Phi}$ into $L_{\Psi}$ and is continuous.

For example, in case $\Phi(u) \sim|u|^{p}$ and $\Psi(u) \sim|u|^{q}$ we get $(\Psi \circ \tilde{\Phi})(u)=(\tilde{\Phi} \circ \Psi)(u) \sim$ $|u|^{p q /(p-1)}$, and so we obtain the special result mentioned above.

A particularly important special case is a weakly singular integral operator of Volterra type

$$
V_{\tau} u(x)=\int_{0}^{x} h(x, y)|x-y|^{-\tau} u(y) d y \quad(0 \leq \tau<1) .
$$

This operator may be considered not only in Lebesgue spaces, but also in the Hölder space $C^{\alpha}$ with norm

$$
\|u\|_{\alpha}:=|u(0)|+\sup _{x \neq y} \frac{|u(x)-u(y)|}{|x-y|^{\alpha}} .
$$

In fact, the following result is classical [8]: 
THEOREM 6. Suppose that

$$
p>\frac{1}{1-\tau}, \quad \alpha \leq 1-\tau-\frac{1}{p} .
$$

Then the operator (6) maps $L_{p}$ into $C^{\alpha}$ and is continuous.

It is natural to ask what happens if the Lebesgue space $L_{p}$ in Theorem 6 is replaced by some Orlicz space $L_{\Phi}$. As one could expect, one has then to replace also the Hölder space $C^{\alpha}$ by some "generalized" Hölder space. Such a space may be constructed as follows (see, e.g., [10]). Given a positive, continuous, increasing function $\phi: \mathbb{R}^{+} \rightarrow \mathbb{R}^{+}$with $\phi(0)=0$, the generalized Hölder space $C^{\phi}$ is defined by the norm

$$
\|u\|_{\phi}:=|u(0)|+\sup _{x \neq y} \frac{|u(x)-u(y)|}{\phi(|x-y|)} .
$$

The following Theorem 7 is taken from [1].

THEOREM 7. Let $f$ and $\phi$ be the scalar functions defined by

$$
f(t)=\int_{0}^{t} \tilde{\Phi}\left(v^{-\tau}\right) d v, \quad \phi(t)=\inf \left\{s>0: f\left(s^{1 / \tau} t\right) \leq s^{1 / \tau}\right\} .
$$

Then the operator (6) maps $L_{\Phi}$ into $C^{\phi}$ and is continuous.

If we take $\Phi(u) \sim|u|^{p}$ in Theorem 7 , we get

$$
\tilde{\Phi}\left(v^{-\tau}\right) \sim|v|^{-\tau p /(p-1)}, \quad f(t) \sim t^{(p-1-\tau p) /(p-1)}, \quad \phi(t) \sim t^{1-\tau-1 / p},
$$

and so we regain precisely Theorem 6 . On the other hand, if $\Phi$ is the second Orlicz function in (1), then

$$
f(t)=\int_{0}^{t}\left(1+v^{-\tau}\right) \log \left(1+v^{-\tau}\right) d v-\int_{0}^{t} v^{-\tau} d v<\infty
$$

and from this we may compute $\phi(t)$ and the target space $C^{\phi}$.

Now we pass to a completely different aspect, viz. to Sobolev imbedding theorems. If $\Omega \subset \mathbb{R}^{N}$ is a bounded domain with sufficiently smooth boundary, the so-called Sobolev conjugate $p^{*}:=N p /(N-p)$ of $p \in[1, N)$ plays a crucial role in such imbedding theorems. In fact, the imbeddings

$$
W^{1, p} \hookrightarrow \begin{cases}L_{p^{*}} & \text { if } p<N \\ L_{q} & (q<\infty) \\ L_{\infty} & \text { if } p=N\end{cases}
$$

are true (see, e.g. [15]), where $W^{1, p}=W^{1, p}(\Omega)$ denotes as usual the Sobolev space consisting of all functions whose first (distributional) derivatives belong to $L_{p}$. Now, one could ask if one of the Lebesgue spaces on the right-hand side of (7) might be replaced by some smaller Orlicz space, in order to make the corresponding imbedding theorem sharper. In fact, the following imbedding theorem [11] makes the second case of (7) more precise and involves the third Orlicz function in (1).

THEOREM 8. In case $p=N$ one has the imbedding $W^{1, N} \hookrightarrow L_{\Psi}$, with $\Psi(u)=e^{|u|^{N /(N-1)}}-1$.

Now we pose the reverse question: Is it possible to replace the Sobolev space $W^{1, p}$ on the left-hand side of (7) by some larger space and still keep the same imbeddings? 
To make this more precise, recall that the Sobolev-Orlicz space $W^{1} L_{\Phi}$ consists of all functions whose first (distributional) derivatives belong to $L_{\Phi}$. The following Theorem 9 was recently proved by Pick [18].

TheOREM 9. Among all Sobolev-Orlicz spaces, the space $W^{1, p}$ is optimal for the imbedding (7) in case $p>N$, but not optimal in case $p=N$.

We point out that the problem of finding an optimal Orlicz function $\Phi$ such that $W^{1} L_{\Phi} \hookrightarrow L_{\Psi}$, with $\Psi(u)=e^{|u|^{N /(N-1)}}-1$, was also solved by Pick in [17].

One may also imitate the imbedding result (7) directly by introducing some kind of Sobolev conjugate of an Orlicz function, rather then a number. Given an Orlicz function $\Phi$, one puts

$$
g(t):=\frac{\Phi^{-1}(t)}{t^{1+1 / N}}, \quad\left(\Phi^{*}\right)^{-1}(v)=\int_{0}^{v} g(t) d t .
$$

The Orlicz function $\Phi^{*}$ is then called Sobolev conjugate to $\Phi$. Let us check what this means in case $\Phi(u) \sim|u|^{p}$ with $1 \leq p<N$. An easy calculation shows that then

$$
g(t) \sim t^{(N-N p-p) / N p}, \quad\left(\Phi^{*}\right)^{-1}(v) \sim|v|^{(N-p) / N p}, \quad \Phi^{*}(u) \sim|u|^{N p /(N-p)},
$$

i.e., we get the classical Sobolev conjugate $p^{*}$, as one should expect. Now we distinguish two cases for the function $g$ in (8), viz.

$$
\int_{0}^{1} g(t) d t<\infty, \quad \int_{1}^{\infty} g(t) d t=\infty .
$$

and

$$
\int_{0}^{1} g(t) d t=\infty, \quad \int_{1}^{\infty} g(t) d t<\infty .
$$

TheOREM 10. The imbedding $W^{1} L_{\Phi} \hookrightarrow L_{\Phi *}$ holds in case (9), while the imbedding $W^{1} L_{\Phi} \hookrightarrow L_{\infty}$ holds in case (10).

We have already seen that $\Phi^{*}(u) \sim|u|^{p^{*}}$ for $\Phi(u) \sim|u|^{p}$ in case (9), i.e. $p<N$, and so Theorem 10 contains the first imbedding in (7) as a special case. Similarly, condition (10) corresponds then to the case $p>N$, and so we get the third imbedding in (7) as well. In the most interesting case $p=N$ we have $g(t)=1 / t$, and so neither (9) nor (10) is satisfied. However, one may then show directly [15] that the Orlicz function $\Phi(u)=|u|^{N}$ has the Sobolev conjugate $\Phi^{*}(u)=e^{|u|}-|u|-1$.

There is a related problem which is of independent interest and may be stated as follows: Is the Orlicz space $L_{\Phi}$ the union of all Orlicz spaces it contains properly? To answer this question we recall that an Orlicz function $\Phi$ satisfies a $\Delta_{3}$-condition if $\Phi(u) \sim$ $|u| \Phi(u)$. Such an Orlicz function necessarily increases more rapidly than any polynomial function. The following result is due to Welland [20].

THEOREM 11. The equality

$$
\bigcup_{L_{\Psi} \subset L_{\Phi}} L_{\Psi}=L_{\Phi}
$$


holds if $\Phi \in \Delta_{2}$, while the equality

$$
\bigcup_{L_{\Psi} \subset L_{\Phi}} L_{\Psi} \neq L_{\Phi}
$$

holds if $\Phi \in \Delta_{3}$.

We intentionally confined ourselves in this talk to some basic problems where Orlicz spaces arise quite naturally. Clearly, almost all results discussed here have been generalized in various directions. One of the most important extensions is that of non-autonomous Orlicz functions (also called modulars) $\Phi: \Omega \times \mathbb{R} \rightarrow \mathbb{R}$. In this case one gets an Orlicz space (or modular space) equipped with the more complicated norm

$$
\|u\|_{\Phi}=\inf \left\{k>0: \int_{\Omega} \Phi\left(x, \frac{|u(x)|}{k}\right) d x \leq 1\right\} .
$$

The first systematic account of such spaces is the Lecture Notes [16], the recent monograph on such spaces, with a particular emphasis to integral operators, is [6]. The norm (11) may be used to construct new spaces from given ones which are important examples of so-called ideal spaces (see Chapter 2 of [2]).

Instead of considering ordinary integral operators of type (4) in Orlicz spaces, one may also consider the more general class of partial integral operators

$$
L u(x, y)=\int_{T} l(x, y, \xi) u(\xi, y) d \mu(\xi), \quad M u(x, y)=\int_{S} m(x, y, \eta) u(x, \eta) d \nu(\eta)
$$

in so-called Orlicz spaces with mixed norm, see [3]. Surprisingly, such problems are not only of theoretical interest, but admit applications in continuum mechanics, like axially symmetric contact problems in surface mechanics, creeping of non-uniformly aging bodies in fracture mechanics, aerodynamics, elasticity and visco-elasticity; we refer the interested reader to [4].

There is a very important field where Orlicz spaces apply which we did not discuss in this talk, viz. partial differential equations. Whenever one has to deal with equations with rapidly growing coefficients, it is useful to replace Lebesgue spaces by Orlicz spaces, and Sobolev spaces by Sobolev-Orlicz spaces. One of the first examples of such applications is the pioneering paper [9] by Jean-Pierre Gossez.

\section{References}

[1] J. Appell and M. Väth, Weakly singular Hammerstein-Volterra operators in Orlicz and Hölder spaces, Zeitschr. Anal. Anw. 12 (1993), 663-676.

[2] J. Appell and P. P. Zabrejko, Nonlinear Superposition Operators, Cambridge Univ. Press, Cambridge, 1990.

[3] J. Appell, A. S. Kalitvin and P. P. Zabrejko, Partial integral operators in Orlicz spaces with mixed norm, Colloquium Math. 78 (1998), 293-306.

[4] J. Appell, A. S. Kalitvin and M. Z. Nashed, On some partial integral equations arising in the mechanics of solids, Zeitschr. Angew. Math. Mech. 79, 10 (1999), 703-713.

[5] S. Banach, Théorie des opérations linéaires, Warszawa, 1932. 
[6] C. Bardaro, J. Musielak and C. Vinti, Nonlinear Integral Equations and Applications, de Gruyter, Berlin, 2003.

[7] B. Bollobás, Linear Analysis-An Introductory Course, Cambridge Univ. Press, Cambridge, 1990 .

[8] R. Gorenflo and S. Vessella, Abel Integral Equations, Lect. Notes Math. 1461, Springer, Berlin, 1991.

[9] J.-P. Gossez, Nonlinear elliptic boundary value problems for equations with rapidly (or slowly) increasing coefficients, Trans. Amer. Math. Soc. 190 (1974), 163-205.

[10] A. I. Gusejnov and Kh. Sh. Mukhtarov, Introduction to the Theory of Nonlinear Singular Integral Equations, Nauka, Moscow, 1980 (in Russian).

[11] J. A. Hempel, G. R. Morris and N. S. Trudinger, On the sharpness of a limiting case of the Sobolev imbedding theorem, Bull. Austral. Math. Soc. 3 (1970), 369-373.

[12] M. A. Krasnosel'skij, On the continuity of the operator $F u(x)=f(x, u(x))$, Dokl. Akad. Nauk SSSR 77 (1951), 185-188 (in Russian).

[13] M. A. Krasnosel'skij and Ya. B. Rutitskij, Convex Functions and Orlicz Spaces, Fizmatgiz, Moscow, 1958 (in Russian); Engl. transl.: Noordhoff, Groningen 1961.

[14] S. G. Krejn, Yu. I. Petunin and E. M. Semenov, Interpolation of Linear Operators, Nauka, Moscow, 1978 (in Russian); Engl. transl.: Transl. Amer. Math. Soc., Providence, R.I., 1982.

[15] A. Kufner, O. John and S. Fučik, Function Spaces, Noordhoff, Leyden, 1977.

[16] J. Musielak, Orlicz Spaces and Modular Spaces, Lect. Notes Math. 1034, Springer, Berlin, 1983.

[17] L. Pick, Optimal Sobolev embeddings, in: Nonlinear Analysis, Function Spaces and Applications, M. Cianchi and A. Kufner (eds.), Olympia Press, Prague, 1999, 156-199.

[18] L. Pick, Optimal Sobolev embeddings - old and new, in: Function Spaces, Interpolation Theory and Related Topics, M. Cwikel (ed.), de Gruyter, Berlin, 2002, 403-411.

[19] M. M. Rao and Z. D. Ren, Theory of Orlicz Spaces, M. Dekker, New York, 1991.

[20] R. Welland, Inclusion relations among Orlicz spaces, Proc. Amer. Math. Soc. 17 (1966), $135-139$. 